\title{
Câncer de Pênis: Resultados e Importância de uma Campanha de Prevenção
}

\author{
Penis Cancer: Results and Importance of a Prevention Campaign
}

Cáncer de Pene: Resultados e Importancia de una Campaña de Prevención

\author{
Adriano Scalzer Correia ${ }^{1}$ \\ Gustavo Victor Furtado da Silva ${ }^{2}$ \\ Humberto Montoro Chagas ${ }^{3}$ \\ Ítalo Marcelo do Rêgo Nascimento ${ }^{4}$ \\ Maria Helena Correia Lessa ${ }^{5}$ \\ Teodorico Romualdo Costa Júnior ${ }^{6}$
}

\section{Resumo}

Objetivo: Relatar os resultados de uma campanha de combate ao Câncer de Pênis no Estado de Alagoas, avaliando o perfil clínico dos pacientes e a importância de tais campanhas como estratégia de prevenção. Método: Estudo descritivo e transversal, amostra de 154 voluntários do sexo masculino atendidos no Mutirão de Combate ao Câncer de Pênis, realizado no Hospital
Universitário Professor Alberto Antunes (HUPAA), Maceió-AL. Avaliou-se idade e patologia clínica, determinandose média e frequência. Resultados: $\mathrm{O}$ total de voluntários foi de 154 , média de idade de 46,85 $\pm 16,67$ anos. Após exame clínico, foram evidenciados 25 $(16,23 \%)$ casos de fimose; $01(0,65 \%)$ caso de leucoplasia; 82 (53,25\%) casos sem patologia peniana ao exame e 46 $(29,87 \%)$ casos com outras patologias penianas, dentre elas $15(32,61 \%)$ casos

${ }^{1}$ Discente do Curso de Medicina. FAMED- Universidade Federal de Alagoas. (UFAL) Endereço completo: A Campus A. C. Simões. Av. Lourival Melo Mota, s/n. Tabuleiro dos Martins. 57072-900. Maceió - Al, Brasil. E-mail: adrianoscalzer2018@gmail.com

${ }^{2}$ Médico Urologista. Docente Hospital Universitário Professor Alberto Antunes (HUPAA)-UFAL

${ }^{3}$ Médico Urologista. HUPAA-UFAL

${ }^{4}$ Médico. Graduado pela FAMED-UFAL

${ }^{5}$ Técnico - Ensino Profissional . Instituto Federal de Alagoas

${ }^{6}$ Discente do Curso de Medicina FAMED. Universidade Federal de Alagoas

Recebido: Nov/2017 - Aceito: Abr/2018.

Revist. Port.: Saúde e Sociedade. 2018;3(1):628-638. 
de balanopostite, $09(19,56 \%)$ casos com excesso de prepúcio mas boa exposição da glande, $07(15,22 \%)$ casos de HPV, 03 (6,52\%) casos de candidíase, $02(4,35 \%)$ casos de herpes genital, $02(4,35 \%)$ casos de associação de lesões por HPV e Herpes genital, 02 $(4,35 \%)$ casos de aderências balanoprepuciais, sem indicação cirúrgica. Conclusões: $\mathrm{O}$ aumento dos casos de câncer de pênis no Brasil, sobretudo no Nordeste, faz com que este seja considerado um problema de saúde pública. Por ser um dos poucos cânceres que podem ser evitados, é de fundamental importância fazer $\mathrm{o}$ diagnóstico precoce desta patologia. Torna-se necessário, então, intensificar atividades como as campanhas de prevenção, podendo, assim, incrementar estratégias como o autoexame, a adequada higiene íntima, a realização de postectomia e a prevenção de infecções sexualmente transmissíveis.

\section{Descritores: Neoplasias Penianas;}

Prevenção de Doenças; Educação em Saúde.

\section{Abstract}

Objective: To report the results of a campaign to combat Penile Cancer in the State of Alagoas, evaluating the clinical profile of the patients and the importance of such campaigns as a prevention strategy. Method: A descriptive and cross-sectional study, a sample of 154 male volunteers attending the Fight Against Penile Cancer, performed at the Professor Alberto Antunes University Hospital (HUPAA), Maceió-AL. Age and clinical pathology were evaluated, and mean and frequency were determined. Results: The total number of volunteers was 154, mean age of 46,85 $\pm 16,67$ years. After clinical examination, 25 $(16.23 \%)$ cases of phimosis were detected; $01 \quad(0.65 \%)$ case of leukoplakia; $82(53.25 \%)$ cases without penile disease on examination and 46 (29.87\%) cases with other penile diseases, among them 15 (32.61\%) cases of balanoposthitis, 09 (19.56\%) cases with excess foreskin but good glans exposure 07 (15.22\%) cases of HPV , $03(6.52 \%)$ cases of candidiasis, $02(4.35 \%)$ cases of genital herpes, 02 (4.35\%) cases of HPV and Herpes genital, 02 (4.35\%) cases of balanopreputial adhesions, without surgical indication. Conclusions: The increase in cases of penile cancer in Brazil, especially in the Northeast, makes it a public health problem. Because it is one of the few cancers that can be avoided, it is of fundamental 
importance to make an early diagnosis of this pathology. It is necessary, then, to intensify activities as prevention campaigns, and can thus increase the strategy such as self-examination, intimate hygiene, a postectomy and prevention of sexually transmitted infections.

\section{Descriptors: Penile Neoplasms;} Disease Prevention; Health Education.

\section{Resumen}

Objetivo: Informar los resultados de una campaña de combate al Cáncer de Pene en el Estado de Alagoas, evaluando el perfil clínico de los pacientes y la importancia de tales campañas como estrategia de prevención. Método: Estudio descriptivo y transversal, muestra de 154 voluntarios del sexo masculino atendidos en el Mutirão de Combate al Cáncer de Pene, realizado en el Hospital Universitario Profesor Alberto Antunes (HUPAA), Maceió-AL. Se evaluó edad y patología clínica, determinándose media y frecuencia. Resultados: El total de voluntarios fue de 154, media de edad de 46,85 $\pm 16,67$ años. Después del examen clínico, se evidenciaron 25 (16,23\%) casos de finósis; 01 (0,65\%) caso de leucoplasia;
$(32,8 \%)$ casos de patopenia peneana al examen y $46(29,87 \%)$ casos con otras patologías penianas, entre ellas 15 (32,61\%) casos de balanopostitis, (19,56\%) casos con exceso de prepucio pero buena exposición del glande, 07 $(15,22 \%)$ casos de HPV, 03 (6,52\%) casos de candidiasis, 02 (4,35\%) casos de herpes genital, 02 (4,35\%) casos de $H P V$ de asociación de lesiones por HPV y Herpes genital, 02 (4,35\%) casos de adherencias balanoprepuciales, sin indicación quirúrgica. Conclusiones: El aumento de los casos de cáncer de pene en Brasil, sobre todo en el Nordeste, hace que éste sea considerado un problema de salud pública. Por ser uno de los pocos cánceres que pueden ser evitados, es de fundamental importancia hacer el diagnóstico precoz de esta patología. Es necesario, entonces, intensificar actividades como las campañas de prevención, pudiendo, así, incrementar estrategias como el autoexamen, la adecuada higiene intima, la realización de postectomía y la prevención de infecciones sexualmente transmisibles.

Descriptores: Neoplasias del Pene; Prevención de Enfermedades; Educación em Salud.

\section{Introdução}


O Câncer de Pênis (CP) é um problema de saúde pública, principalmente em países em desenvolvimento, sendo rara e com incidência decrescente em países desenvolvidos, atingindo aproximadamente $\quad 1 / 100.000$ homens $^{(1,2)}$, com frequência de $0,24 \%$ dos cânceres que acometem o homem nos Estados Unidos $^{(3)}$ e $0,4 \%$ na Europa $^{(3,4)}$.

A baixa incidência desta doença nos países desenvolvidos, em contraste com a alta incidência nos países em desenvolvimento, indica a associação da doença com perfil socioeconômico de cada país ${ }^{(1,2,4,5,6,10)}$.

No Brasil, o CP representa 2\% das neoplasias do sexo masculino, atingindo índices de 5,7\% na região Nordeste, $5,3 \%$ na região Norte, $3,8 \%$ na região Centro-Oeste, $1,4 \%$ na região Sudeste e $1,2 \%$ na região $\mathrm{Sul}^{(1,10)}$, sendo que nas regiões de maior prevalência, o número de casos superou casos de cânceres de próstata e bexiga $^{(4,5,6,10)}$ (Gráfico 1).

Gráfico 1 - Frequência Relativa de Câncer de Pênis por Região do Brasil

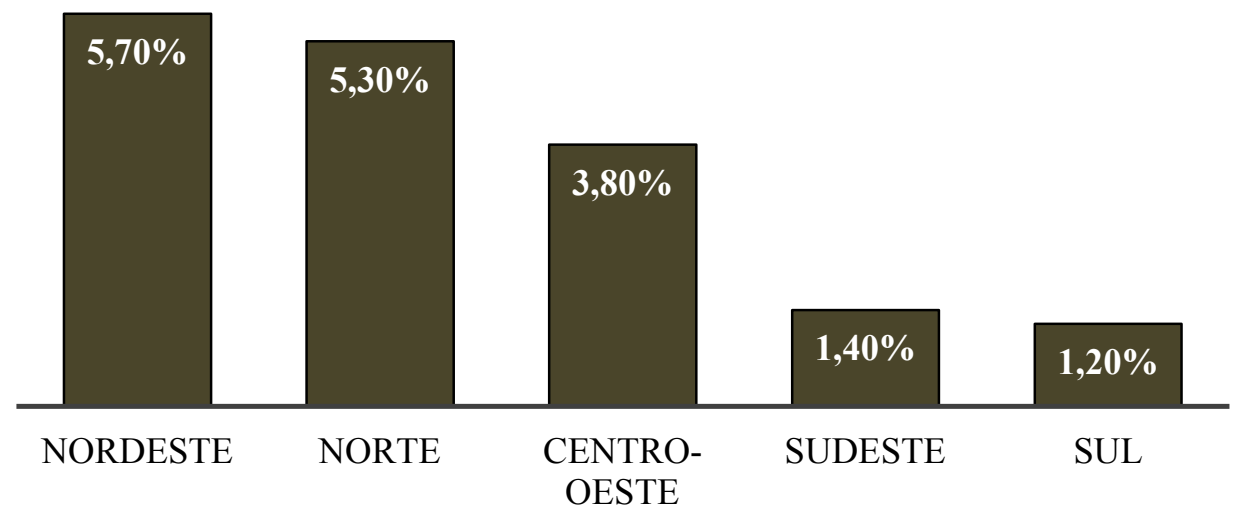

$\square$ Frequência

Acomete, em geral, homens entre a quinta e a sexta década de vida ${ }^{(6)}$, apresentando diversos fatores de risco. Homens que possuem fimose ou excesso de prepúcio, de baixo nível socioeconômico e falta de higiene pessoal tem os fatores de risco mais importantes para o desenvolvimento de câncer de pênis ${ }^{(1)}$.

Homens com fimose carregam um risco aumentado de $25 \%$ a $60 \%$ para câncer de pênis. Outros fatores de risco 
incluem balanite, inflamação crônica, trauma peniano, o uso do tabaco, líquen escleroso, e histórico de doenças sexualmente transmissíveis, especialmente o papilomavírus humano $(\mathrm{HPV})^{(7-12)}$.

Estudos demonstram que, em geral, de $45 \%$ a $80 \%$ dos cânceres penianos estão relacionados com HPV, com uma forte correlação com os tipos 16 e $18^{(7)}$. Já outros afirmam que o DNA do HPV está presente em 40\% a $45 \%$ dos casos de carcinoma peniano ${ }^{(9)}$ e alguns detectaram o HPV em 30,5\% dos casos, sendo $52,0 \%$ do tipo $16^{(12)}$. Os fumantes são considerados 3,0 a 4,5 vezes mais propensos a desenvolver câncer de pênis ${ }^{(7)}$.

O tratamento da neoplasia é predominantemente cirúrgico, com retirada da lesão e, em alguns casos, com a amputação parcial do membro e a retirada dos gânglios da região inguinal (primeiro sítio de metástase dessa doença). O diagnóstico precoce é fundamental para evitar 0 desenvolvimento da doença e a amputação, que acarretam consequências físicas, sexuais e psicológicas para o paciente ${ }^{(1)}$.

Devido à carência de dados e estudos sobre o tema em nosso Estado, este trabalho visa, primeiramente, relatar os resultados de uma campanha de combate ao Câncer de Pênis realizada no Estado de Alagoas. Além disso, avaliar o perfil clínico dos pacientes desta campanha, fazendo um paralelo com dados nacionais e internacionais, e, também, avaliar a importância de tais campanhas como estratégia de prevenção.

\section{Método}

Em novembro de 2015, acompanhando o movimento internacional que busca a conscientização a respeito do câncer de próstata e da saúde masculina, no Brasil conhecido com o nome de Novembro Azul, a Sociedade Brasileira de Urologia, Secção Alagoas, realizou o Mutirão de Combate ao Câncer de Pênis.

Esta campanha ocorreu no Hospital Universitário Professor Alberto Antunes (HUPAA), MaceióAL, na data de 28 de novembro de 2015, de 8 às 14h. Participaram do evento Médicos Urologistas, a Liga Urológica de Alagoas (UFAL), a Liga Acadêmica de Radiologia e Diagnóstico por Imagem (UFAL) e a Liga Urológica Acadêmica da Uncisal. A divulgação para comparecimento dos voluntários se deu por meio de panfletos e convite 
através de mídia televisiva (Jornal local).

Os atendimentos ocorreram em ambulatório, no qual foi preenchida uma ficha de cadastro, seguido do exame clínico para identificação de lesão sugestiva de câncer de pênis ou de fator de risco como fimose. Conforme os resultados do exame físico, os pacientes foram orientados a realizar exames pré-operatórios e tratamento cirúrgico no HUPAA (casos de fimose).

Após a realização da campanha, produziu-se um relatório com todos os dados e informações devidamente colhidos, cujo título é "Relatório da Campanha - Mutirão de Combate ao Câncer de Pênis". Este documento encontra-se de posse da Sociedade Brasileira de Urologia, Secção Alagoas, sendo de livre acesso ao público interessado.

Foi realizado, então, estudo descritivo e transversal de um grupo de 154 voluntários do sexo masculino, cujos dados foram extraídos e adaptados do Relatório da Campanha - Mutirão de Combate ao Câncer de Pênis, fornecido pela Sociedade Brasileira de Urologia, Secção Alagoas. Avaliou-se idade e patologia clínica, determinando média e frequência. Uma cópia do referido relatório também encontra-se de posse do autor principal deste manuscrito para consultas.

Este estudo não requer parecer de Comitê de Ética - CEP-CONEP, conforme Art. $1^{\circ}$, incisos II, III e V, da Resolução $\mathrm{N}^{\mathrm{o}}$ 510, de 07 de abril de 2016, que dispõe sobre as normas aplicáveis a pesquisas em Ciências Humanas e Sociais, uma vez que trata de pesquisa envolvendo apenas dados secundários, de domínio público e que não identificam os participantes da pesquisa.

\section{Resultados}

O total de voluntários foi de 154 no Mutirão de Combate ao Câncer de Pênis, com média de idade de 46,85 anos (DP: $\pm 16,67)$, sendo a idade mínima de 4 anos e a idade máxima de 81 anos.

Após realização de exame clínico, dos 154 pacientes, foram evidenciados $25(16,23 \%)$ casos com indicação de cirurgia/postectomia (casos de fimose), $01(0,65 \%)$ caso com indicação de biópsia (leucoplasia); $82 \quad(53,25 \%)$ casos sem patologia peniana ao exame e $46 \quad(29,87 \%)$ casos com outras patologias penianas (Tabela 1). 
Tabela 1 - Frequência de Patologias Penianas Após Exame Clínico

\begin{tabular}{lr}
\hline Patologia peniana & \multicolumn{1}{c}{ Casos N (\%) } \\
\hline Fimose & $25(16,23 \%)$ \\
Leucoplasia & $01(0,65 \%)$ \\
Ausência de patologia peniana & $82(53,25 \%)$ \\
Outras patologias penianas & $46(29,87 \%)$ \\
Total & $154(100 \%)$ \\
\hline
\end{tabular}

*HPV: Papilomavírus humano

Dentre os 46 casos de patologias penianas, a distribuição ocorreu da seguinte forma: $15(32,61 \%)$ casos de balanopostite, $09(19,56 \%)$ casos com excesso de prepúcio, mas boa exposição da glande, 07 (15,22\%) casos de HPV,
$03(6,52 \%)$ casos de candidíase, 02 $(4,35 \%)$ casos de herpes genital, 02 $(4,35 \%)$ casos de associação de lesões por HPV e Herpes genital, 02 (4,35\%) casos de aderências balanoprepuciais, sem indicação cirúrgica (Tabela 2).

Tabela 2 - Frequência de Outras Patologias Penianas

\begin{tabular}{lr}
\hline Outras Patologias Penianas & \multicolumn{1}{c}{ Casos N (\%) } \\
\hline Balanopostite & $15(32,61 \%)$ \\
Excesso de prepúcio, boa exposição da & $09(19,56 \%)$ \\
glande & \\
HPV* & $07(15,22 \%)$ \\
Candidíase & $03(6,52 \%)$ \\
Herpes genital & $02(4,35 \%)$ \\
HPV e herpes genital & $02(4,35 \%)$ \\
Aderências balanoprepuciais & $02(4,35 \%)$ \\
Outras & $06(13,04 \%)$ \\
Total & $46(100 \%)$
\end{tabular}

*HPV: Papilomavírus humano 


\begin{abstract}
Casos com indicação de postectomia foram orientados $\mathrm{e}$ encaminhados para realização de exames pré-operatórios e tratamento cirúrgico no Hospital Universitário Professor Alberto Antunes (HUPAA).
\end{abstract}

\section{Discussão}

A partir da análise de estudos, constatou-se que, para o câncer de pênis, há uma concordância em relação aos fatores de risco para $o$ desenvolvimento da neoplasia. Como principais fatores de risco, do ponto de vista epidemiológico, encontramos a não-realização de circuncisão, a presença de fimose, a higiene inadequada, as infecções virais e o comportamento sexual de risco $^{(1-16)}$. Porém, ressalta-se que há uma associação estreita com questões socioeconômicas. Fatores como baixa escolaridade, má instrução e higiene íntima inadequada ocupam papel de destaque quando analisamos o perfil clínico, cultural e epidemiológico desses pacientes ${ }^{(4)}$.

Segundo Clark PE et $\mathrm{al}^{(7)}$, homens com fimose carregam um risco aumentado de $25 \%$ a $60 \%$ para câncer de pênis. No presente trabalho, verificamos um número representativo de casos apresentando fimose $(16,23 \%)$ e que tiveram indicação de cirurgia/postectomia. A correção cirúrgica torna-se fundamental nestes casos, pois o acúmulo de secreções na glande (esmegma), associada a presença de Mycobaterium smegatis, além de causar irritação crônica do epitélio, contribui para a gênese do câncer de pênis ${ }^{(10)}$

A presença de fimose e, também, o excesso de prepúcio $(19,56 \%$ dos casos) estão intimamente relacionados à higiene inadequada, já que podem dificultar a exposição e, assim, a correta higienização da glande, predispondo o indivíduo às infecções.

Foram encontrados, dentre os 46 casos com patologias penianas, 15 (32,61\%) casos de balanopostite, que é a inflamação conjunta da glande e do prepúcio. A inflamação desta região pode ser provocada por diversos fatores, como hábitos higiênicos inadequados, mas também pelo uso de agentes irritativos na limpeza do pênis, sendo aconselhável o uso de produtos neutros (por exemplo: sabão neutro). As causas infecciosas, entretanto, merecem atenção especial, já que podem estar relacionadas a comportamento sexual de risco. 
Outro fator de risco importante são as infecções virais, sobretudo a infecção pelo papilomavírus humano, sendo que, na literatura, a taxa de infecção por este tipo de vírus em câncer de pênis varia de $20 \%$ a $75 \%{ }^{(3)}$. Nestes estudos a detecção se deu por diferentes métodos sorológicos, o que justifica em parte a variabilidade dos resultados encontrados. Em nosso trabalho foram observados, dentre aqueles com patologias, 07 (15,22\%) casos de HPV. Número, este, bastante expressivo, visto que se trata de casos já com presença de lesões detectáveis ao simples exame físico.

Vale lembrar que muitos estudos epidemiológicos demonstram estreita correlação entre câncer de colo uterino e carcinoma peniano. Mulheres cujos parceiros apresentam câncer de pênis possuem risco 2,8 a 3,2 vezes mais elevado para o desenvolvimento do câncer de colo uterino ${ }^{(10)}$. Estes achados revelam, portanto, o HPV como agente etiológico do carcinoma de cérvice uterina, tornando provável a associação entre o HPV e o câncer de pênis ${ }^{(1,2,10)}$.

Estão, ainda, envolvidos na etiologia do câncer de pênis outras infecções virais, como o herpes genital, presente em $02(4,35 \%)$ dos casos aqui analisados, as infecções fúngicas, candidíase estava presente em 03
$(6,52 \%)$ dos casos e as associações, sendo que em 02 (4,35\%) casos havia associação de lesões por HPV e Herpes genital.

Finalmente, através da análise dos dados, verificamos que as principais patologias encontradas na campanha, apenas com exame clínico e, por isto, de baixo custo, correspondem aos principais fatores de risco preveníveis associados à neoplasia peniana demonstrados na literatura utilizada.

\section{Conclusão}

Dados da Secretaria de Estado da Saúde (SES) alertam que, além do câncer de próstata, o câncer de pênis atinge a população masculina de forma dramática. Apesar de ser um tumor raro, exige, muitas vezes, a amputação do órgão. O aumento dos casos de câncer de pênis no Brasil, sobretudo no Nordeste, superando inclusive o número de casos de câncer de próstata e de bexiga, faz com que este seja considerado um problema de saúde pública.

Conforme revela alguns estudos, este é um dos poucos cânceres que podem ser evitados, sendo de fundamental importância fazer $\mathrm{O}$ diagnóstico precoce. Campanhas de prevenção como esta podem, então, 
diagnosticar o câncer de pênis logo nos estágios iniciais, proporcionando maiores chances de cura, aumento da sobrevida, como também reduzir a incidência e a severidade da doença.

Torna-se necessário intensificar este tipo de atividade, veiculando à população em geral o conhecimento sobre esta patologia, como, por exemplo, a associação dos maus hábitos de higiene e o efeito carcinogênico da fimose e da infecção pelo HPV. Podese, assim, incrementar as estratégias de prevenção, primeiramente com medidas simples e eficazes, como o autoexame e a adequada higiene íntima. Somado a isso, a realização de postectomia, nos casos em que houver indicação, e a prevenção de infecções sexualmente transmissíveis.

Devido à carência de informações sobre o tema em nosso Estado, este trabalho representa uma importante fonte de dados para a realização de futuros estudos sobre o Câncer de Pênis no Estado de Alagoas.

\section{Referências}

1. Favorito LA, Nardi AC, Ronalsa M, Zequi SC, Sampaio FJB, Glina S. Epidemiologic study on penile cancer in Brazil. Urology (Rio de Janeiro) [periódico na internet]. 2008 [citado 2015 dez. 14]; 34(5): 587-93. Disponível em: http://www.scielo. br/scielo.php?script $=$ sci_arttext\&pid $=\mathrm{S}$ 1677-55382008000500007.
2. Reis AAS et al. Aspectos clínicoepidemiológicos associados ao câncer de pênis. Ciênc saúde coletiva. 2010; 15(Supl. 1): 1105-11.

3. Spiess PE, Dhillon J, Baumgarten AS, Johnstone PA, Giuliano AR. Pathophysiological basis of human papillomavirus in penile cancer: key to prevention and delivery of more effective therapies. American Cancer Society (Atlanta) [periódico na internet]. 2016 [citado 2015 dez. 21]; 66(6): 481-95. Disponível em: https://onlinelibrary.wiley.com/doi/full /10.3322/caac.21354.

4. Souza VC, Dourado SMM. Câncer de pênis no Brasil: um problema de saúde pública. Oncologia (São Paulo) [periódico na internet]. 2015 [citado 2015 dez. 23]; 11(40): 58-9. Disponível em: https://www.sboc.org .br/sboc-site/revista-sboc/pdfs/40/edito rial.pdf.

5. Instituto Nacional de Câncer. Disponível em: http://www.inca.gov.br

6. Carvalho NS, Kannenberg AP, Munaretto C, Yoshioka D, Absy MCV, Ferreira MA, Filho RT. Associação entre HPV e câncer peniano: Revisão da literatura. Doenças Sexuamente Transmissíveis (Niterói) [periódico na internet]. 2007 [citado 2015 dez. 26]; 19(2): 92-5. Disponível em: http:// www.dst.uff.br//revista19-2-007/6.pdf.

7. Clark PE et al. Penile cancer: clinical practice guidelines in oncology. Oncology (Washington) [periódico na internet]. 2013 [citado 2016 jan. 14]; 11(5): 594-15. Disponível em: https://www.ncbi.nlm. nih.gov/pubmed/23667209\#.

8. Dillner J, von Krogh G, Horenblas $\mathrm{S}$, Meijer CJ. Etiology of squamous cell carcinoma of the penis. Scand J Urol Nephrol Suppl. 2000; 205: 18993. 
9. Gross G, Pfister H. Role of human papillomavirus in penile cancer, penile intraepithelial squamous cell neoplasias and in genital warts. Med Microbiol Immunol. 2004; 193(1): 3544.

10. Costa S, Rodrigues R, Barbosa L, Silva JO, Brandão JOC, Medeiros, CSQ. Câncer de pênis: epidemiologia e estratégias de prevenção. Ciências Biológicas (Recife) [periódico na internet]. 2013 [citado 2016 jan. 21]; 1(2): 23-3. Disponível em: https://periodicos.set.edu.br/index.php/ facipesaude/article/view/1197.

11. Pow-Sang MR, Benavente V, Pow-Sang JE, Morante C, Meza L Baker M, Pow-Sang JM. Cancer of the penis. Canc Contr J. 2002; 9(4): 30514.

12. Bezerra AL et al. Human papillomavirus as a prognostic factor in carcinoma of the penis: analysis of 82 patients treated with amputation and bilateral lymphadenectomy. Oncology [periódico na internet]. 2001 [citado 2016 jan. 27]; 91(12): 2315-21. Disponível em: https://www.ncbi.nlm. nih.gov/pubmed/11413520.

13. Souza KW, Reis PED, Gomes IP, Carvalho EC. Estratégias de prevenção para câncer de testículo e pênis: revisão integrativa. Enfermagem (São Paulo) [periódico na internet]. 2001 [citado 2016 jan. 31]; 2011; 45(1): 277-82. Disponível em: http://www .scielo.br/scielo.php?script=sci_arttext \&pid=S0080-62342011000100039.
14. Pizzocaro G, Algaba F, Horenblas S, Solsona E, Tana S, Van Der Poel H, Watkin N. Diretrizes para o câncer de pênis. Urologia (Rio de Janeiro) [periódico na internet]. 2010 [citado 2016 fev. 1]; 57(6): 1002-12. Disponível em: http://portaldaurologia. org.br/medicos/wp-content/uploads/20 17/06/98.pdf.

15. Mosconia AM, Roilaa F, Gattab $\mathrm{G}$, Theodore C. Cancer of the penis. Critical Reviews in Oncology/Hematology. 2005; 53: 165 77.

16. Pompeo ACL. Câncer de pênis: um problema brasileiro. Urologia (Rio de Janeiro) [periódico na internet]. 2017 [citado 2017 jun. 30]. Disponível em: http://portaldaurologia.org.br/do encas/cancer-de-penis-um-problemabrasileiro/. 